\title{
Antenna radiation patterns indication on the basic measurement of field radiation in the near zone
}

\author{
M. Wnuk \\ Faculty of Electronics, Military University of Technology, Poland
}

\begin{abstract}
The paper presents the method of antennas radiation patterns measurements in the near zone. Afterwards, using the analytical methods, the measured data is transformed into radiations patterns in the far field. This technique is used in measurements in the closed areas, which enable researchers to manage the environmental characteristics. The calculated radiation patterns are as precise as the range measurements in the far field. It needs to be outlined however that this method requires more complex and expensive regulation procedures as well as more sophisticated software, whereas the radiation patterns are not obtained in the real time.
\end{abstract}

Keywords: antennas radiation, near zone, far field.

\section{Introduction}

An antenna is one of the important components of a radio communication system. It is designed to convert the input current into an electromagnetic field and to emit it into the surrounding space (transmitting antenna) to the contrary (receiving antenna). Therefore, the antenna is a device which adjusts the waveguide to free space. Due to its location between a transmitting or receiving device and the space, requirements set forth for an antenna are imposed both by the conditions of expansion of electromagnetic waves in space and by interaction of the antenna as an element of the given device on its operation [1].

Its parameters and patterns affect not only effective information transfer but also meeting the compatibility conditions, i.e. the antenna should not disturb operation of other systems, particularly along the lateral lobe radiation lines. That is why during the recent period the antenna measurement technique 
develops very rapidly. It must ensure high accuracy of measurements, which must be taken at a receding level of signal. This results from the necessity to measure lateral lobes at below $-40 \mathrm{~dB}$. In order to meet these requirements the measurements should be taken in special conditions, which are ensured in anechoic chambers.

\section{Antenna radiation zones}

The area surrounding the antenna may be typically split into three zones of the electromagnetic field generated by that antenna: the reactive near-field, the radiating near-field, the far-field (Figure 1). The far-field expands to infinity and it is that area in space in which the electromagnetic field changes with distance $r$ from the transmitting antenna according to the $\exp (-j k r) / r$ relationship, where $k=$ $2 \pi / \lambda, \lambda$ - wave length.

It is assumed that the far-field expands between the distance from the studied antenna to infinity, where $D$ is the largest geometrical dimension of the studied antenna.

$$
R_{g}=\left(\frac{2 D^{2}}{\lambda}\right)+\lambda
$$

Factor $\lambda$ added to the $2 D^{2} / \lambda$ relationship covers the case in which the maximum geometrical dimension of the antenna is smaller than wave length $\lambda$. The space area stretching between the antenna and the conventional limit of the far-field is called the near-field, with the reactive near-field expanding between the studied antenna and distance $\lambda$. In the reactive near-field the electric and magnetic field phases are almost in quadrature, the Poynting vector is of a complex nature.

The imaginary part of the Poynting vector is responsible for collecting the energy of the electromagnetic field near the antenna surface, and the real part relates to energy emitted by the antenna.

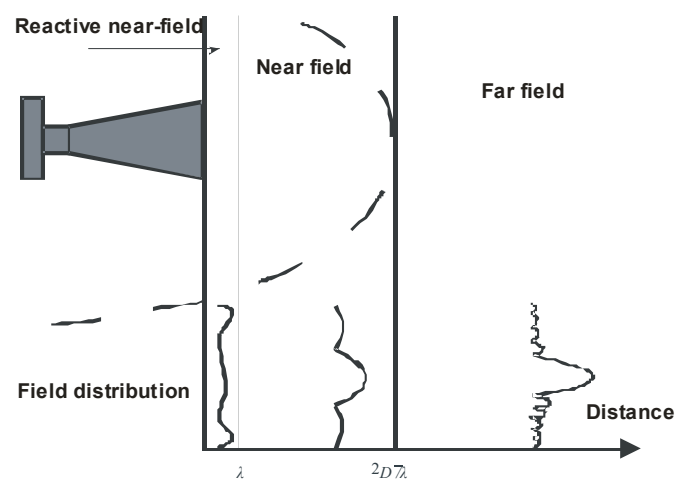

Figure 1: Zones around the antenna.

At a distance greater than $\lambda$ from the antenna the electromagnetic field has a complex nature and changes significantly in the function of distance from the 
antenna, it is the so-called radiating near-field. Antenna measurements in the near-field are usually taken in the radiating near-field. Sometimes areas of the electromagnetic field around the antenna are named with terms borrowed from optics, such as the Fresnel zone and Fraunhofer zone. The Fraunhofer zone is a term equivalent with the far-field, whereas the Fresnel zone expands from distance to the conventional limit of the far-field [1].

$$
R_{d}=\left(\frac{D}{2 \lambda}\right)^{\frac{1}{3}}\left(\frac{D}{2}\right)+\lambda
$$

Traditional methods require the measurements to be taken in the far-field.

It is difficult to meet this requirement in case of antennas operating in the micro wave range. For instance, the radius of this zone, for an $L=3 \mathrm{~m}$ antenna with working frequency of $f=9 \mathrm{GHz}$, expands to a distance of over $540 \mathrm{~m}$. It should be noted that the size of the largest anechoic chambers does not exceed $50 \mathrm{~m}$. Hence the need to develop such methods which allow reduction of the size of the measured structure do dimensions suitable for confined spaces such as an anechoic chamber.

This problem may be solved by taking measurements in the near-field the socalled $(4 \div 10) \lambda$ distance.

There are many methods of measurements in the near-field. Three of them are generally used. These are: the planar method (Figure 2(a)), the cylindrical method (Figure 2(b)) and the spherical method (Figure 2(c)). Each of the methods has both advantages and disadvantages. Spherical scanning requires larger anechoic chambers, as compared with the remaining methods.

Cylindrical scanning proves excellently for measurement of area monitoring radars. Planar scanning is limited by the angular sector which allows measurement of the main beam and the nearest lateral lobes. The main advantage of planar scanning consists in dense and uniform distribution of sampling points in the grid. In the case of scanning polar (uniaxial) and bipolar (biaxial) plane it will be possible to obtain a scanning plane larger than that offered by the anechoic chamber.

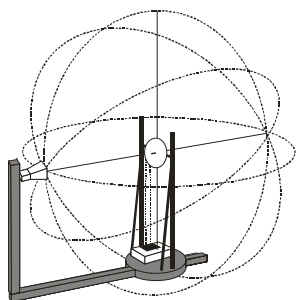

(a) spherical method

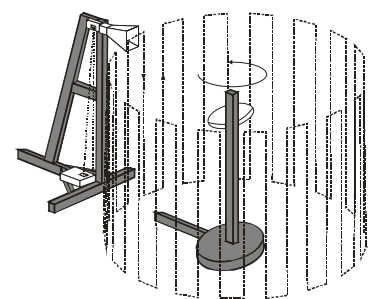

(b) cylindrical method

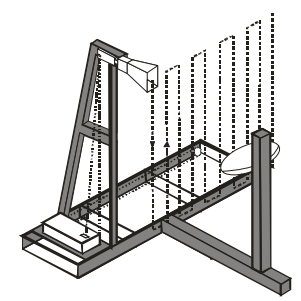

(c) rectangular planar method

Figure 2: View of setups for antenna measurements in the near-field.

In the methods of antenna measurement in the near-field, the values of the electromagnetic field are measured at discrete points in a preset surface. Sampling points are in nodes of suitably defined grid, inscribed on this surface. 
Three types of sampling point grids are applied; they are presented in Fig. 3. Excessively dense sampling is not necessary for accurate presentation of the electromagnetic field. In practice, a ca $10-20 \%$ redundancy coefficient is introduced. Redundant density of sample occurrence is mostly affected by the pattern in which the lines with sampling points converge into a single central point (3(b)), (3(c)). Such patterns of sampling point grids are often used in practice due to the advantageous kinematic systems of the scanner.

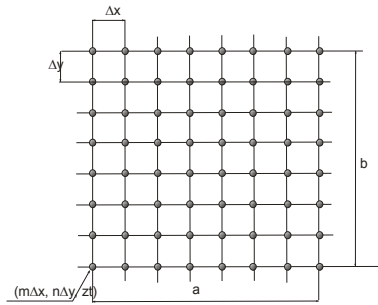

(a) Rectangular grid

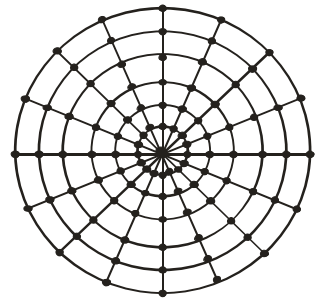

(b) Uniaxial planar grid

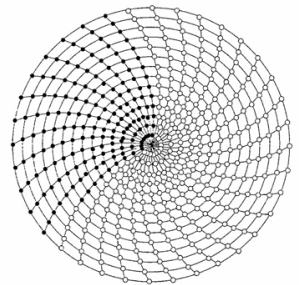

(c) Biaxial planar grid

Figure 3: $\quad$ Measurement grids.

The radiating area of the near-field expands between the distance equal to $\lambda$ wave length from the antenna and the distance determined by formula (1). Beyond this distance we have the far-field, where angular distribution of energy does not oscillate with the distance and the radiating power disappears with the distance. Dimension of the measurement area is important, because we are considering accuracy of the planar measurement technique in the near-field.

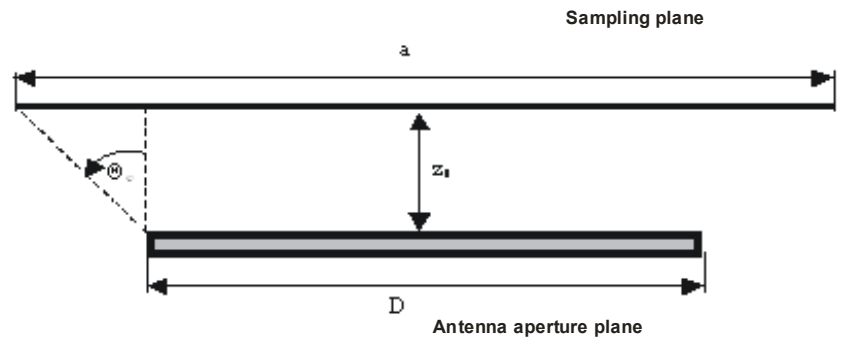

Figure 4: Clarification of the size of angular sector of the area of importance of the measured pattern.

The size and location of the measurement area define the value of the angular sector of the area of importance. The size of this angular sector depends, i.a., on the size of scanning surface of the electromagnetic field and on the distance of this surface from the aperture of the tested antenna [7]. The computed radiation pattern in the far-field will be precise in the $\pm \Theta_{S}$ area.

$$
\Theta_{S}=\operatorname{arctg}\left(\frac{a-D}{2 z_{t}}\right)
$$


Total angular coverage may be obtained in the spherical system by adding measurements in the near-field along the whole spherical surface of the nearfield

Regardless of the chosen measurement method, equipment for carrying out specific measurements is similar. The differences are primarily caused by: arrangement of different measuring instruments in respect of the source of the measurement signal and the tested antenna, the type of measurements to be taken and the required level of automation of measurements.

The equipment required for taking measurements of antenna patterns in the near-field consists of 4 major sub-systems which may be controlled from one, central control panel. These are:

- positioning and control subsystem,

- receiving subsystem,

- $\quad$ signal source subsystem,

- measurement data saving and processing subsystem.

It should be emphasized that while taking measurements in the near-field the results obtained should be transformed, with the use of analytical methods, into data suitable for computing radiation patterns in the far-field. The computed radiation patterns are as accurate as measurement of range in the far-field.

Depending on the required accuracy it is necessary to use more complex and expensive regulatory procedures and more complicated software and radiation patterns are not obtained in real time.

\section{Theoretical basis for determination of antenna pattern based on the near-field measurement}

Modern planar scanning techniques in measurements of the antenna near-field are based on representation of the field in form of planar wave spectrum. Electromagnetic waves with a given frequency may be represented as a superposition of elementary planar waves of the same frequency. Further considerations shall be based on a rectangular $\mathrm{x}, \mathrm{y}, \mathrm{z}$ coordinate system (Figure 5).

In the passive and lossless area of free space, Maxwell equations describing the phenomenon of electromagnetic wave propagation, may be transformed into homogeneous second order Helmholtz equations [9],

$$
\begin{aligned}
& \nabla^{2} \vec{E}+k^{2} \vec{E}=0 \\
& \nabla^{2} \vec{H}+k^{2} \vec{H}=0 \\
& \nabla \cdot \vec{E}=\nabla \cdot \vec{H}=0
\end{aligned}
$$

On assumption that observations of components of the vector of electric and magnetic field, for a wave sinusoidal variable in time, were carried out at the same moment $t=t_{p}$ in all studied points of space, the segments dependent on independent variable $t$ representing time, were abandoned in the above equations.

Due to linearity of the said operators and linearity of the medium in which the described phenomenon of electromagnetic wave propagation takes place, it is 
fairly easy to prove that the equations below satisfy the set of equations (4), (5), (6) and the threshold requirements in plane $z=0$,

$$
\begin{gathered}
\vec{E}(x, y, z)=\int_{-\infty-\infty}^{+\infty} \int_{-\infty}^{+\infty} \vec{A}\left(k_{x}, k_{y}\right) \exp (-j \vec{k} \vec{r}) d k_{x} d k_{y}, \\
\vec{H}(x, y, z)=\int_{-\infty-\infty}^{+\infty} \int_{\vec{k}} \vec{k} \times \vec{A}\left(k_{x}, k_{y}\right) \exp (-j \vec{k} \vec{r}) d k_{x} d k_{y}, \\
\vec{k} \cdot \vec{A}\left(k_{x}, k_{y}\right)=0 .
\end{gathered}
$$

where: $\vec{k}=k_{x} \vec{i}_{x}+k_{y} \vec{i}_{y}+k_{z} \vec{i}_{z}$ - wave vector, indicates the direction of propagation of the wave described by wave equations (4), (5), (6),

$k^{2}=\vec{k} \cdot \vec{k} \quad$ - wave number (it is the length of the wave vector),

$\vec{r}=x \vec{i}_{x}+y \vec{i}_{y}+z \vec{i}_{z} \quad$ - vector indicating to observation point,

$\vec{A}\left(k_{x}, k_{y}\right)=A_{x}\left(k_{x}, k_{y}\right) \vec{i}_{x}+A_{y}\left(k_{x}, k_{y}\right) \vec{i}_{y}+A_{z}\left(k_{x}, k_{y}\right) \vec{i}_{z}$ - wave vector describing the planar wave spectrum.

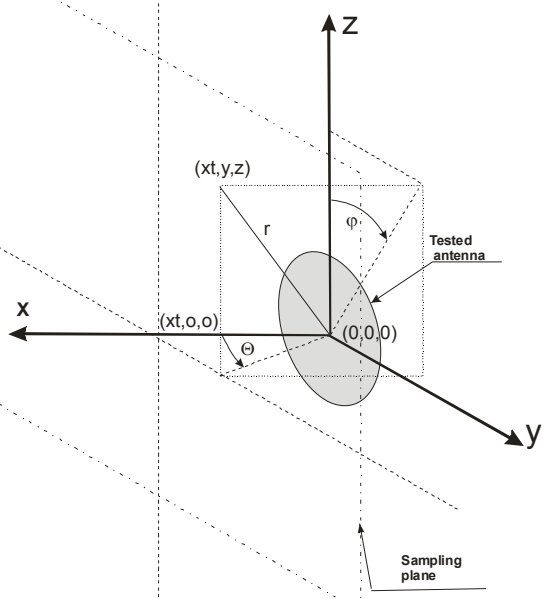

Figure 5: Location of the antenna in the reference arrangement adopted in the analysis

The integrand $\vec{A}\left(k_{x}, k_{y}\right) \exp (-j \vec{k} \vec{r})$ occurring in relationships (7), (9) represents the homogeneous planar wave propagating along the direction determined by vector $\vec{k}$ therefore a monochrome wave emitted through the aperture may be recorded as superposition of planar waves with the same frequency, different amplitudes and expanding in different directions. Equation (9) in turn, which is a natural consequence of the Gauss law for a 
passive area expressed in form of equation (6), allows distinguishing two independent components (here $A_{x}\left(k_{x}, k_{y}\right)$ i $\left.A_{y}\left(k_{x}, k_{y}\right)\right)$ of vector $\vec{A}\left(k_{x}, k_{y}\right)$

$$
A_{z}\left(k_{x}, k_{y}\right)=-\frac{1}{k_{z}}\left(A_{x}\left(k_{x}, k_{y}\right) k_{x}+A_{y}\left(k_{x}, k_{y}\right) k_{y}\right) .
$$

In order to determine the value of the electric field for an aperture located in the far-field, the following relationship was obtained with the use of expression (7),

$$
\vec{E}(r, \theta, \varphi)=\frac{j}{2 \pi} \frac{\exp (-j k r)}{r} k_{z} \vec{A}\left(k_{x}, k_{y}\right)=\frac{j}{2 \pi} \frac{\exp (-j k r)}{r} k_{z}\left(\begin{array}{c}
A_{x}\left(k_{x}, k_{y}\right) \\
A_{y}\left(k_{x}, k_{y}\right) \\
A_{z}\left(k_{x}, k_{y}\right)
\end{array}\right),
$$

where:

$$
\begin{aligned}
& k_{x}=k \sin \theta \cos \varphi, \\
& k_{y}=k \sin \theta \sin \varphi, \\
& k_{z}=k \cos \theta, \\
& A_{z}\left(k_{x}, k_{y}\right) \text { is expressed by (10). }
\end{aligned}
$$

The necessity to determine components $E_{\theta}=(r, \theta, \varphi)$ and $E_{\varphi}=(r, \theta, \varphi)$ of the far-field determined in spherical coordinates implies carrying out transformations which produce the relationship (11) in respective form:

$$
\begin{aligned}
& \vec{E}(r, \theta, \varphi)=\frac{j k}{2 \pi} \frac{\exp (-j k r)}{r}\left(\left(A_{x}\left(k_{x}, k_{y}\right) \cos \varphi+A_{y}\left(k_{x}, k_{y}\right) \sin \varphi\right) i_{\theta}+\right. \\
& \left.+\cos \theta\left(A_{y} \cos \varphi-A_{x} \sin \varphi\right) i_{\varphi}\right)=E_{\theta}\left(k_{x}, k_{y}\right) i_{\theta}+E_{\varphi}\left(k_{x}, k_{y}\right) i_{\varphi}
\end{aligned}
$$

In the next step, depending on the method of polarisation of the tested aperture, we determine co-polarisation $E_{c o}(\theta, \varphi)$ and cross-polarisation $E_{\text {cross }}(\theta, \varphi)$ patterns.

Polarization of antenna $E_{x}$ :

$$
\begin{aligned}
& E_{c o}(\theta, \varphi)=E_{\theta}(\theta, \varphi) \cos \varphi-E_{\varphi}(\theta, \varphi) \sin \varphi= \\
& =A_{x}\left(k_{x}, k_{y}\right)\left(\cos ^{2} \varphi+\sin ^{2} \varphi \cos \theta\right)+A_{y}\left(k_{x}, k_{y}\right) \sin \varphi \cos \varphi(1-\cos \theta) \\
& E_{\text {cross }}(\theta, \varphi)=E_{\theta}(\theta, \varphi) \sin \varphi-E_{\varphi}(\theta, \varphi) \cos \varphi= \\
& =A_{x}\left(k_{x}, k_{y}\right) \sin \varphi \cos \varphi(1-\cos \theta)+A_{y}\left(k_{x}, k_{y}\right)\left(\cos ^{2} \varphi+\cos \theta \cos ^{2} \varphi\right)
\end{aligned}
$$

plane $E(\varphi=0)$ :

$$
\begin{aligned}
E_{c o}(\theta, \varphi) & =A_{x}\left(k_{x}, k_{y}\right), \\
E_{\text {cross }}(\theta, \varphi) & =A_{x}\left(k_{x}, k_{y}\right) \cos \theta,
\end{aligned}
$$

plane $H\left(\varphi=\frac{\pi}{2}\right)$ :

$$
\begin{gathered}
E_{c o}(\theta, \varphi)=A_{x}\left(k_{x}, k_{y}\right) \cos \theta \\
E_{\text {cross }}(\theta, \varphi)=A_{x}\left(k_{x}, k_{y}\right) .
\end{gathered}
$$

In order to determine the far-field pattern it is necessary to know the components $A_{x}\left(k_{x}, k_{y}\right)$ and $A_{y}\left(k_{x}, k_{y}\right)$ of the planar wave spectrum vector $\vec{A}\left(k_{x}, k_{y}\right)$. In the 
case of observation of the electric field vector in the $z=z_{t}$ plane, the vector equation (7) adopts the following form:

$$
\begin{aligned}
& E_{x}\left(x, y, z=z_{t}\right)=\int_{-\infty-\infty}^{+\infty} \int_{x}^{+\infty}\left[A_{x}\left(k_{x}, k_{y}\right) \exp \left(-j k_{z} z_{t}\right)\right] \exp \left(-j k_{z} x\right) \exp \left(-j k_{z} y\right) d k_{x} d k_{y} \\
& E_{y}\left(x, y, z=z_{t}\right)=\int_{-\infty-\infty}^{+\infty+\infty}\left[A_{y}\left(k_{x}, k_{y}\right) \exp \left(-j k_{z} z_{t}\right)\right] \exp \left(-j k_{z} x\right) \exp \left(-j k_{z} y\right) d k_{x} d k_{y} \\
& E_{z}\left(x, y, z=z_{t}\right)=\int_{-\infty-\infty}^{+\infty+\infty}\left[A_{z}\left(k_{x}, k_{y}\right) \exp \left(-j k_{z} z_{t}\right)\right] \exp \left(-j k_{z} x\right) \exp \left(-j k_{z} y\right) d k_{x} d k_{y}
\end{aligned}
$$

where:

$$
k_{z}=\left\{\begin{array}{ccc}
\sqrt{k^{2}-k_{x}^{2}-k_{y}^{2}} & \text { for } & k_{x}^{2}+k_{y}^{2} \leq k^{2} \\
\sqrt{-j\left(k^{2}-k_{x}^{2}-k_{y}^{2}\right)} & \text { for } & k_{x}^{2}+k_{y}^{2}>k^{2}
\end{array}\right.
$$

Selection of sample spacing allows obtaining such equations for Fourier integrals in which the structure is actually a modified version of two-dimensional discrete Fourier transform or inverse. Because of the considerable number of sampling points acquired during scanning the measurement plane, the choice of effective numerical algorithms for data processing becomes essential. A good example here is offered by algorithms of Fourier fast transform (FFT) and inverse Fourier fast transform (IFFT), which may be applied to determine transforms based on line/column type decomposition.

For a finite number of observation points ( $2 N$ sampling points) and the rectilinear domain $(s \in(-\Delta s, \Delta s))$ the expression may be as follows:

$$
\widetilde{F}(s)=\sum_{n=-N+1}^{N} \frac{\sin w(s-n \Delta s)}{w(s-n \Delta s)} \psi(s-n \Delta s) \vec{F}(n \Delta s) .
$$

The approximating function $\psi(s)$ is designed to ensure quick convergence of the approximation error with the growing value of the rate of oversampling $\chi=\frac{\pi / w}{\Delta s}(\pi / w$ is the maximum admissible spacing - Nyquist spacing resulting from the sampling theorem) and minimisation of the so-called truncation error resulting from the finite size of the measurement grid.

On the other hand, in the case of occurrence of approximation of angular variable domain $\varphi(\varphi \in(-\Delta \varphi, \Delta \varphi))$ in the task, the following rule should be applied:

$$
\widetilde{F}(\varphi)=\sum_{m=-M+1}^{M} D_{M_{n}}(\varphi-m \Delta \varphi) \Omega_{M_{r}}(\varphi-m \Delta \varphi) \vec{F}(m \Delta \varphi),
$$

where:

$$
D_{M_{n}}(\varphi)=\frac{\sin \left[\left(2 M_{n}+1\right) \frac{\varphi}{2}\right]}{\left(2 M_{n}+1\right) \sin (\varphi / 2)}-\text { Dirichlet function, }
$$


$\Omega_{M_{r}}(\varphi, 0)$ plays the role of a function which reduces the truncation error value.

In view of the fact that this paper describes the application of planar scanning, errors for this case shall not be analysed.

\section{Principles of field sampling in the near-field}

In this paper the antenna was tested with the use of the planar method with a rectangular grid of sample points. This choice was based upon its major advantages, such as: low cost of scanning mechanism, the smallest amount of computations and stationary tested antenna.

Data acquisition in a planar near-field is done over a rectangular $x-y$ grid, Figure 3(a), with maximum sample spacing in the near-field

$$
\Delta x=\Delta y=\lambda / 2
$$

The measurement procedure requires that the $z_{t}$ plane surface at a distance from the tested antenna be selected where the measurements are taken. The $z_{t}$ distance should be located at a distance of at least two or three wavelengths between the tested antenna and the near-field interaction limit.

The plane in which the measurements are taken is split into a rectangular grid with $M \times N$ points spaced $\Delta x$ and $\Delta y$ apart and defined by coordinates $\left(m \Delta x n \Delta y, z_{t}\right)$, where:

$$
-\frac{M}{2} \leq m \leq \frac{M}{2}-1 \quad \text { and } \quad-\frac{N}{2} \leq n \leq \frac{N}{2}-1
$$

Values $M$ and $N$ are determined by linear dimensions of sampling plane divided by sampling spaces. Measurements are taken till the time when the signal at the plane edges reaches the level of $-40 \mathrm{~dB}$ below the highest level of the signal inside the measured plane. Defining $a$ and $b$ as the width and height for the measured plane, $M$ and $N$ are determined by the expressions:

$$
M=\frac{a}{\Delta x}+1 \quad \text { and } \quad N=\frac{b}{\Delta y}+1
$$

The selected sampling spaces in the measurement grid should be smaller than half the wave length and should meet the Nyquist sampling criterion. If plane $z=$ $z_{t}$ is located in the far-field of the source, sampling spaces may grow to its maximum value $\lambda / 2$. Points of the rectangular grid are spaced by grid spacing, as:

$$
\Delta x=\frac{\pi}{k_{x o}} \quad \text { and } \quad \Delta y=\frac{\pi}{k_{y o}}
$$

where $k_{x o}$ and $k_{y o}$ are real numbers and are the largest dimensions $k_{x}$ and $k_{y}$ respectively, so that $f\left(k_{x}, k_{y}\right) \cong 0$ for $\left|k_{x}\right|>k_{x o}$ or $\left|k_{y}\right|>k_{y o}$. 


\section{Verification of the experiment}

Determination of the radiation pattern of the antenna based on data from measurements taken in the near-field requires application of an advanced mathematical tool. Because of complicated computing techniques, large volumes of input data, the requirement of graphical interpretation of computation results, the Matlab 6.5 programme was used. Two computer programmes were developed. The first of them determines theoretical distributions of the electric field on the surface of the antenna aperture, as well as distributions of intensity and phase of the electric field in a plane in parallel with the aperture plane at $z_{t}$ distance from it. The second programme determines the cross-section of the radiation pattern of the tested parabolic antenna based on data from measurements or theoretical data determined by the first programme.

In order to check the correctness of the developed concept for measurement setup concept antenna measurements were carried out with a symmetrical dish reflector with diameter $D=0.6 \mathrm{~m}$. The radiant element used was in form of halfwave dipole, combined with a circular convergent mirror.

In this case the far-field will occur at the distance of $7.2 \mathrm{~m}$. The size of the available anechoic chamber allow measurements at a maximum distance of $5 \mathrm{~m}$. The figures below present measurements of the amplitude and electromagnetic field phase pattern of the tested antenna, measured in the near-field. They were taken in an anechoic chamber. The amplitude and signal phase were measured with the use of vector analyzer HP HP8530 with accuracy to two decimal places. The results of measurements were standardized. The reference was adopted as the $0 \mathrm{~dB}$ signal level. The phase difference was computed by a frequency converter based on the signal received from the antenna to the reference signal ratio. Figures 6 and 7 present the measured distributions of electric field amplitudes in the scanning plane for component $E_{x}$.

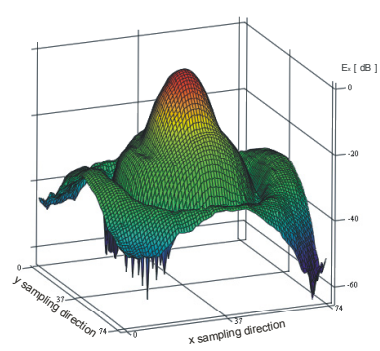

Figure 6: Spatial standardized amplitude pattern of the tested antenna in the near-field.

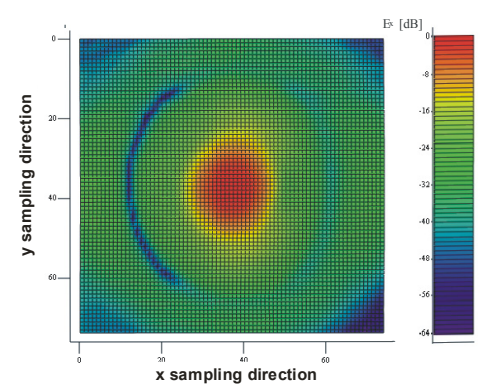

Figure 7: Standardized amplitude of electric field intensity.

Whereas Figure 8 presents the measured distribution of the electric field phase in the scanning plane. 
The kierunkowa programme was used for determination of the following patterns: theoretical (based on data generated by the pole_bliskie programme) and that obtained from computations (based on formula (11). Both patterns are presented in one figure - Figure 9 .

The same figure also additionally presents radiation pattern obtained from measurements. In such diagram comparisons and verification of the experiment may be done.

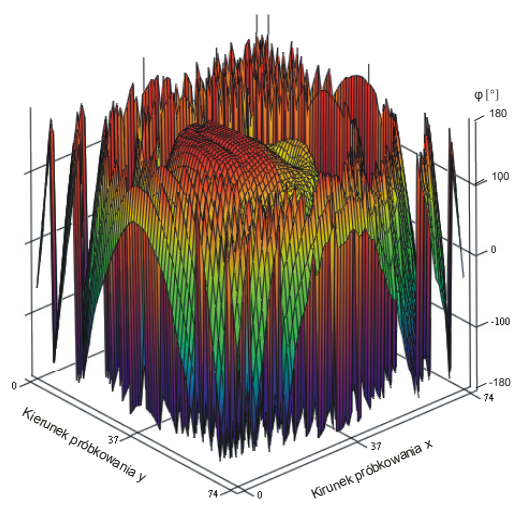

Figure 8: Spatial phase pattern of the tested antenna in the near-field.

The patterns have been marked with numbers and colours respectively:

- Blue - cross-section of the antenna radiation pattern, determined from measurements - No 1;

- Red - cross-section of the antenna radiation pattern computed by the kierunkowa programme based on data received from the antenna nearfield - No 2;

- Green - theoretical cross-section of the antenna radiation pattern generated by the pole_bliskie and kierunkowa programmes - No 3.

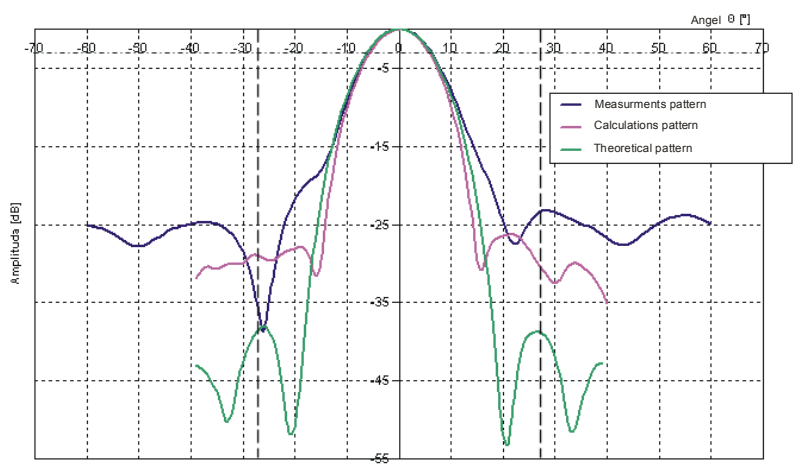

Figure 9: Radiation patterns of the tested antenna. 
While comparing the patterns of Figure 9, one may observe contraction of patterns 2 and 3 as compared with 1 . This is caused not only by phase disturbances but also by failure to meet the requirement of the far-field during measurements of pattern No 1. The difference between the far-field limit and the distance at which the measurements were taken and which amounts to $2.85 \mathrm{~m}$ is so significant that it has a direct impact on the pattern form.

\section{Conclusions}

It was assumed in the to-date analysis that components tangent to the measurement plane of the electric field vector are measured precisely at point. In reality such probe does not exist and the antenna used for measurements has some definite geometrical dimensions. Therefore the values of the amplitude and phase are averaged on its surface. The impact of the probe radiation pattern is also significant.

The pattern was further distorted by imprecise, non-automatic scanning setup. Precision of positioning the probe in vertical plane is not satisfactory, nor is the time necessary for completion of measurements.

The measurement results obtained confirmed the correctness of adopted design assumptions and correctness of algorithms made. Radiation patterns transformed into the far-field are convergent with the theoretical patterns and the results of comparative measurements.

\section{References}

[1] R. E. Collin; Prowadzenie fal elektromagnetycznych; WNT Warszawa 1966,

[2] HP 8530A Microwave Receiver. Operating and Programming Manual, Edition 2, Hewlett-Packard Company, February 1994.

[3] P. Kabacik: Reliable evaluation and property determination of modern-day advanced antennas; Oficyna Wydawnicza Politechniki Wrocławskiej, Wrocław 2004.

[4] J. Modelski, E. Jajszczyk, H. Chaciński, P. Majchrzak: Pomiary parametrów anten Oficyna Wydawnicza Politechniki Warszawskiej, Warszawa 2004.

[5] Y. Rahmat-Samii, L. I. Williams, and R.G. Yaccarino: „The ULCA bi-polar planar near-field antenna -measurement and diagnostic range", IEEE. Antennas and Propagat., Magazine., vol. 37, pp. 16-34, December, 1995.

[6] H. Trzaska; Pomiary pól elektromagnetycznych w polu bliskim. PWN 1998,

[7] W Zieniutycz; Anteny. Podstawy polowe; WKŁ Warszawa 2001.

[8] http://www.nearfield.com

[9] M. Wnuk; Analiza struktur promieniujacych położonych na wielowarstwowym dielektryku, Wojskowa Akademia Techniczna, Warszawa 1999 